\title{
Towards a Semantic Layer Design for an Advanced Intelligent Multimodal Transportation System
}

\author{
Jihane LARIOUI ${ }^{1}$, Abdeltif EL BYED ${ }^{2}$ \\ Laboratory of computer science and modelling of decision support systems \\ HASSAN 2 University Faculty of Science Ain Chock \\ Casablanca, Morocco \\ ${ }^{1}$ jihane.larioui-etu@etu.univh2c.ma \\ 2 abdeltif.elbyed@univh2c.ma
}

\begin{abstract}
Over time, intelligent transport systems (ITS) have shown their relevance and have become essential for good urban mobility management within smart cities. They facilitate traffic management and make flow more fluid and autonomous. Indeed, the data manipulated in the ITS is large and diversified which makes the information less exploitable and leads us to a considerable loss of time before obtaining the desired information. In order to manage these different sources of information, their semantic conflicts and ensure interoperability between all these sources involved in the multi-modal transport network, we decided to integrate all this information in a way that it is unified, flexible and understandable by all the agents of our multimodal information system. Thus, the objective of this semantic approach is to localize the different resources of the WEB, to standardize the description models so that they are usable by the different agents of the system. In this article, we propose a new approach for the development of an architecture based on Multi-Agent systems (SMA) coupled with semantic Web and this in order to help decision-making and improve the management in the context of urban mobility. The system to develop covers all modes of transport and capable of managing planned trips as well as answering questions related to safety during travel.
\end{abstract}

Key words: Multi-agent System (SMA), Intelligent transport systems (ITS), Ontology, Semantic Web, Multi-modal Transportation

\section{INTRODUCTION}

Nowadays, more attention is given to urban mobility due to the increase in population density, traffic congestion, lack of information and all the problems encountered by the transport network. The complexity of traveling in a multimodal network increases over time due to the lack of accurate knowledge and necessary information. Hence the need to develop an intelligent solution that will facilitate decision-making and route planning. Moreover, a semantic approach can be effective in overcoming, sharing knowledge and communication between the different agents of our system.

In multimodal transport, users use at least two different types of transport to reach their destination. Therefore, multimodality refers to the optimal use of different modes of transport. Hence, the need for a system providing real-time information on departures, routes and traffic conditions before and during the travel. In such a situation, the passenger must manage a variety of information: different modes of transport that can be combined (bus, train, metro, etc.), travel time, cost, connection points, different operators, and so do we. In order to manage these different sources of information, their semantic conflicts and ensure interoperability between all these sources involved in the multi-modal transport network, we decided to integrate all this information in a way that it is unified, flexible and understandable by all the agents of our multimodal information system.

Usually, most research contributions consider a multi-agent system to improve route planning in a multimodal network, aside from that, they have not considered using the Semantic Web to allow flexible querying information.

The global purpose of the advanced multimodal information system is to propose the optimal route that corresponds to the different criteria of preferences chosen by the passenger according to their needs in terms of: travel time, number of mode changes, cost and safety. This system is based on a multi-agent system architecture coupled with semantic layer approach for a better management of the multimodal transport network. The purpose of the Semantic layer is to characterize existing information in order to facilitate the automation of services, to discover, link and infer similar knowledge. Its architecture is based on a hierarchy that aims to represent knowledge on the web by satisfying the criteria of standardization, interoperability and flexibility. 
With this in mind, the system to develop is based on an ontology of public transport and should be capable of reasoning. In this article, we detail the semantic layer of our system that acts as middleware between the physical layer and the information layer. In addition, the semantic layer is used to efficiently integrate data and information into our system, also reduce uncertainty in the decision-making process and resolve semantic conflicts resulting from cooperation between different sources.

The structure of this paper is as follows: Section 2 discusses some related works and the role of the ontology in a multi-agent system. The approach used for ontology development and design are stated in section 3. Section 4 describes the Implementation part and discusses the results. Finally, we conclude the document with some remarks and perspectives.

\section{BACKGROUND}

\subsection{Related Works}

In the literature, agent-based information systems are commonly referred to as Advanced Traveler Information Systems (ATIS). The multi-agent system has been applied to various problems related to the transport system. In particular, planning personalized itineraries. Meeting the needs and preferences of users has recently become a major objective of ITS.

Wang, Ding and Jiang [4] have developed an ontology based public transport query system to provide an efficient and timely information service. They opted for an improved public transport request algorithm. This algorithm takes into account both user needs and traffic conditions. They were interested in solving the problem of the same station name in different places to find the shortest distance between two places using a radius search algorithm. Houda et al. [6] developed a domain based transportation system for passenger planning system. The system supports a multi-model journey pattern, which helps the user to find the most convenient path from one position to another with several possible options. Choice of the user depends on interesting journey patterns, shopping journey pattern, etc. The system is limited to the roadways and railways only.

Otherwise, Zgaya et al. [14] have implemented a negotiation protocol for the transport sector. This protocol is based on a flexible ontology to facilitate communication between agents. The work of Niaraki and Kim [16] is focused on determining an impedance model for the road geographic information system and the intelligent transport system. Impedance is a model that aims to calculate the amount of cost or resistance, of the link between a node of origin and that of destination. For this, they defined an ontology of the road segment based on user preference criteria and environmental context criteria, and from this ontology, they created a hierarchical structure divided in two: a part linked to the criteria of users (such as information on tourist attractions and preferences) and the other to contextual criteria (such as weather and safety). This multi-criteria study is carried out using the AHP
(Analytical Hierarchical Process), which consists of defining weights to calculate the impedance. The related works mentioned above give an idea of a domain-based transport ontology to manage the planning of trips between two sites. Related work only offers systems to meet travel needs. However, they do not take into account user preferences.

In all these related works, no formal semantics describing all aspects relating to intelligent road transport systems have been proposed. In this article, we present a transport system ontology for a sensitive semantic transport system which covers all modes of transport and which is capable of managing travel planning as well as answering questions related to transport cost, travel time, route, number of modes changes and safety, etc.

\subsection{The Role of the ontology in a multi-agent system}

The word ontology first appeared in Aristotle's philosophical essays, where he described the nature and organization of being. In artificial intelligence, an ontology is used to represent a domain of knowledge.

The definition of ontology as a semantic primitive is simply a hierarchical description of the important concepts in a domain, coupled with a description of each of these concepts [17]. The ontologies consist of several concepts that include class, subclass, class hierarchy, instance, location, value, default value, facet, type, cardinality, inheritance, variable and relation [18].

The main functionalities of ontology are to share a generic understanding of the structure of information between human and / or software agents, to allow the reuse of domain knowledge, to make domain hypotheses more precise, to create a semantics of interoperability between different information resources, to provide a clear and exact analysis of terms [7].

The objective of this work is to implement these advantages to set up a semantic layer to model the different sources of data, to standardize their description models so that they are comprehensible and usable by the various agents of the system.

Indeed, in a multi-agent system, agents are led to recognize and understand the different concepts describing the application of the domain. These concepts are explicitly defined by ontologies and are associated to the agent with their relationships and constraints [8]. Interoperability between agents is achieved by unifying these concepts using common ontologies that allow agents to cooperate while retaining their autonomy. In Multi-agent systems, agents are linked to various information resources. These information must be effectively integrated into the system so that semantic conflicts are reduced. The most effective way to remedy this problem is to set up a global ontology that will be used by all agents. Agents sharing the same ontology can exchange knowledge and communicate fluently. In fact, their knowledge representations are compatible with respect to the concepts considered relevant and with respect to the names given to these concepts [4]. In this way, the decision-making process will be established in better conditions. 


\section{SEMANTIC LAYER FOR ITS}

\subsection{Global Architecture}

Faced with the complexity of the data manipulated in the ITS systems which are large and diversified [21], we have proposed a novel approach, which combines between Semantic Web technologies and multi-agents paradigm to design an advanced multi-agent information system for multimodal transportation. It would be an effective way to communicate and coordinate between the different agents and a way to improve decision-making process. Previous work for the development of an advanced and intelligent multimodal information system has been proposed to develop a new architecture based on multi-agent systems. This architecture consists of a six layers which are IHM layer, Selection Layer, Decision Making Layer, Information Layer, Semantic Layer and physical layer [18] and [21].(Fig.1)

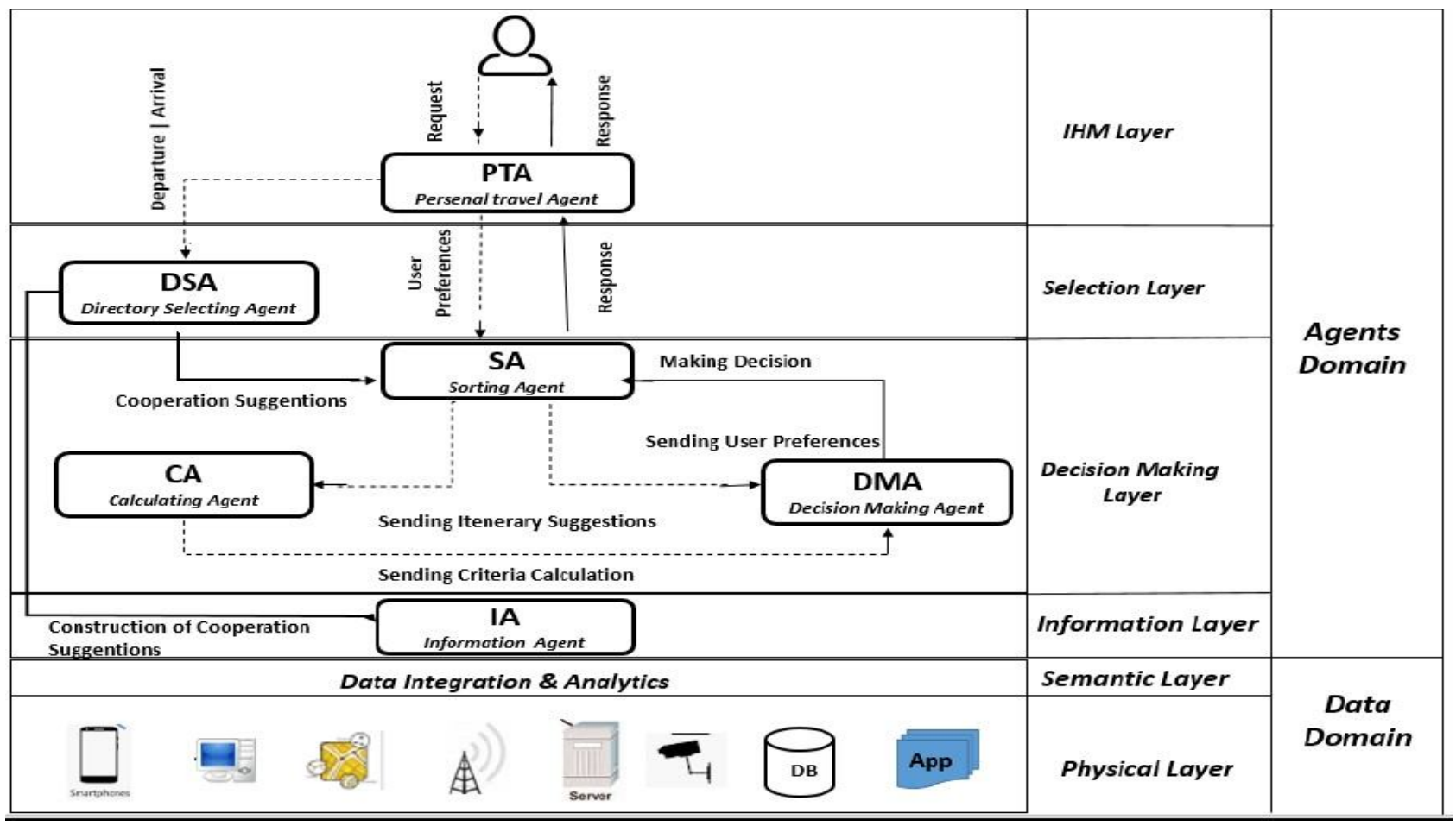

Figure 1: Multi-Agent Information System Architecture

The IHM layer is composed of the PTA "Personal Travel Agent" representing the Human Machine Interface.

- The Selection Layer is composed of The DSA "Directory Selecting Agent", which defines the search domain by specifying the information agents that will work together to plan the route and propose the different combinations of possible information agents.

- The Decision-making layer is composed of three agents: ○ The SA "Sorting Agent", which examines the different routes proposed by the DSA and decides how to treat them according to the preferences of the users.

- The DMA "Decision Making Agent" which is based on the method TOPSIS "Technique for order of preference by similarity to ideal solution" as a MCDM methodology to facilitate decision-making and choose the itinerary that will satisfy the user's preferences.

- The CA "Calculating Agent", which takes up the routes proposed by the DSA in order to calculate for each route its necessary parameters. These parameters are calculated based on user preferences (travel time, number of mode changes, cost and safety).

- The Information Layer is composed of the IA "Information Agents", which are responsible for searching, collecting, integrating and manipulating the information from the different sources of information.

- The Semantic layer, which uses semantics web technology to improve the flexibility between the different agents of the system, in different terms; the semantic layer act like a middleware between the physical layer and the information layer. In addition, the semantic layer is used to efficiently integrate data and information in our system, also reduce the uncertainty in the decision-making process and to resolve the semantic conflicts generated from the cooperation between the different sources.

- The physical layer, which encapsulates multiple information resources, for example: the databases of the different transport operators (Tramway, metro, bus...etc), the transport network architecture, road ITS applications, Sensors, surveillance camera...etc. These systems produce information on urban networks and are essential when the passenger is moving because they provide multimodal information and facilitate the use of networks. 


\subsection{Semantic Layer}

Going back to the semantic layer, the global role of this layer consists of four main functionalities: The first concerns the connectivity to information resources in order to have access to the different data sources. This first step then allows the extraction of the most relevant concepts and the elimination of the miss understanding between these information [22]. Then comes the most important step, which allows generating standard and unified models for these data sources and finally to have a flexible Data Model understandable by all the agents of the system. (Fig.2)

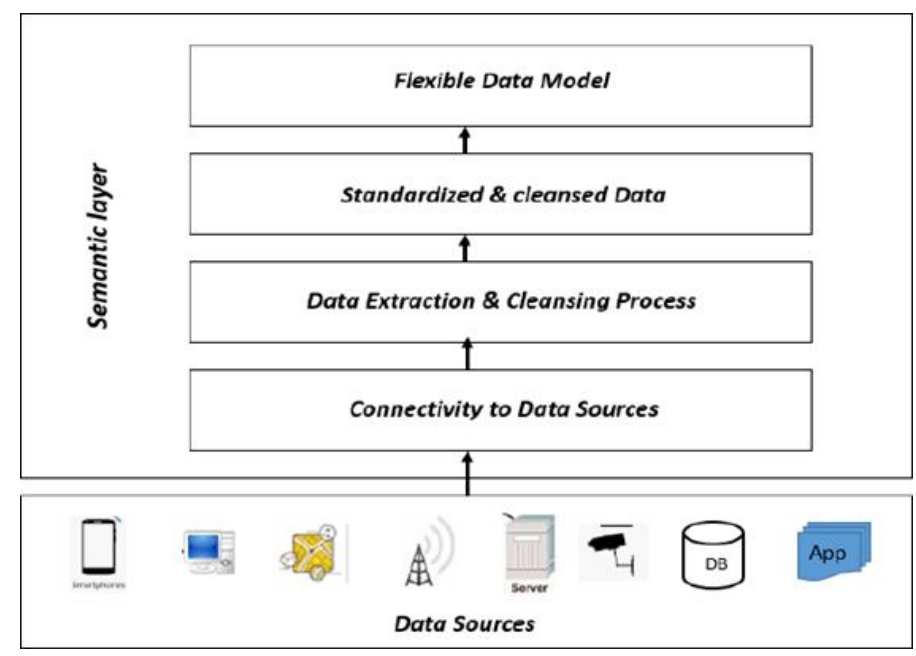

Figure 2: Semantic Layer Process Description

The purpose of this semantic layer process is to formulate all data and concepts contributing to the semantic definition of the multimodal transport system and to standardize the description models, so that they are comprehensible and usable by all agents.

\subsection{Ontology Design}

In this section, we present the ontology design methodology, which is based on a hybrid architecture proposing a bottom up process to create global ontology from local ontologies and define the relationships between them [19].

The proposed solution avoids misunderstandings that can arise during the exchange and communications between agents and aims to make them able to understand each other when using this information processed by these ontologies. We use Protégé 5.1.0 [13] to build our ontology as it is a free open source editor and framework and it supports the latest OWL 2 Web Ontology Language and RDF specifications from the World Wide Web Consortium.

As Fig. 3 shows, the development of the ontology includes three phases:

- The first phase: consists of building local ontologies. Foremost, it is essential to analyze the different information resources, each independently. After that, we define the concepts considered relevant for each ontology, their relationships and their usage constraints.
- The second phase: after having defined the local ontologies, now, we can pull out the global ontology from different defined concepts. This phase includes two stages: first, the analysis of local ontologies, then the selection of all concepts and the resolution of semantic conflicts in order to define the global ontology and its particular concepts.

- The third phase: it consists of defining the mapping between global and local ontologies. The global ontology is constructed from local ontologies using OWL Annotations.

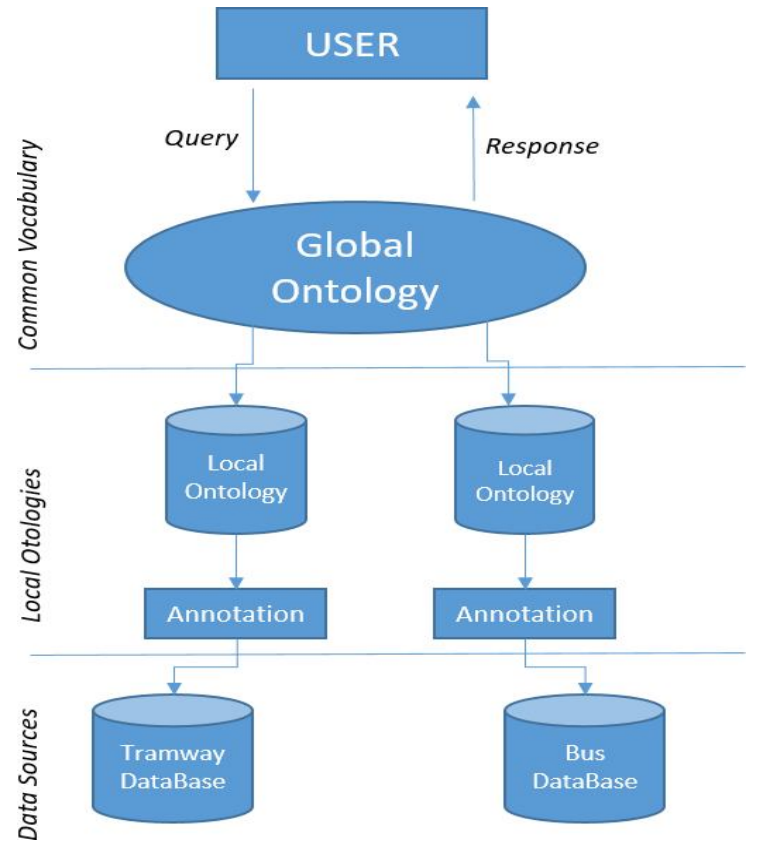

Figure 3: Process of building Ontologies trough 3 steps

\section{IMPLEMENTATION AND EXPERIMENTAL RESULTS}

In this section, we focus on the implementation part. First, we detail the first phase, which concerns the construction of local ontologies and we present only the cases of two information resources, those of the bus and the tramway. The global ontology of the multimodal transport network take up the different concepts and relationships of the local ontologies that we have created: Tramway Ontology and Bus Ontology. The figures below respectively show the local ontologies built based on the data from Bus and Tramway network. The ontologies created use the modeling of the transport network for each mode by illustrating major concepts such as lines, stations, operator, vehicle ...etc. (Fig.4, Fig.5, Fig.6 and Fig.7). By analyzing these concepts, we notice that each ontology defines its concepts differently, for example, the concept Station defined in the Tramway ontology is equivalent to Bus Stop in Bus ontology. In the other hand, we have concepts that have the same definition, for example, Timetable or Operator, also the notion of Vehicles and Lines. 

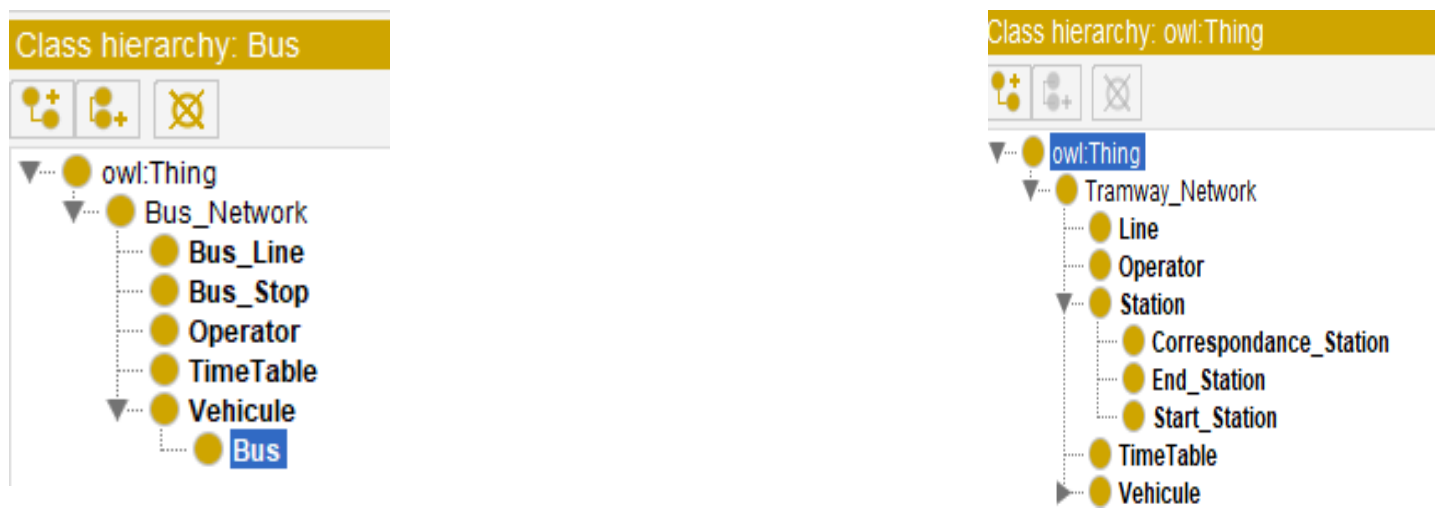

Figure 4: Fragment of Bus Network local Ontology

Figure 5: Fragment of Tramway Network local Ontology

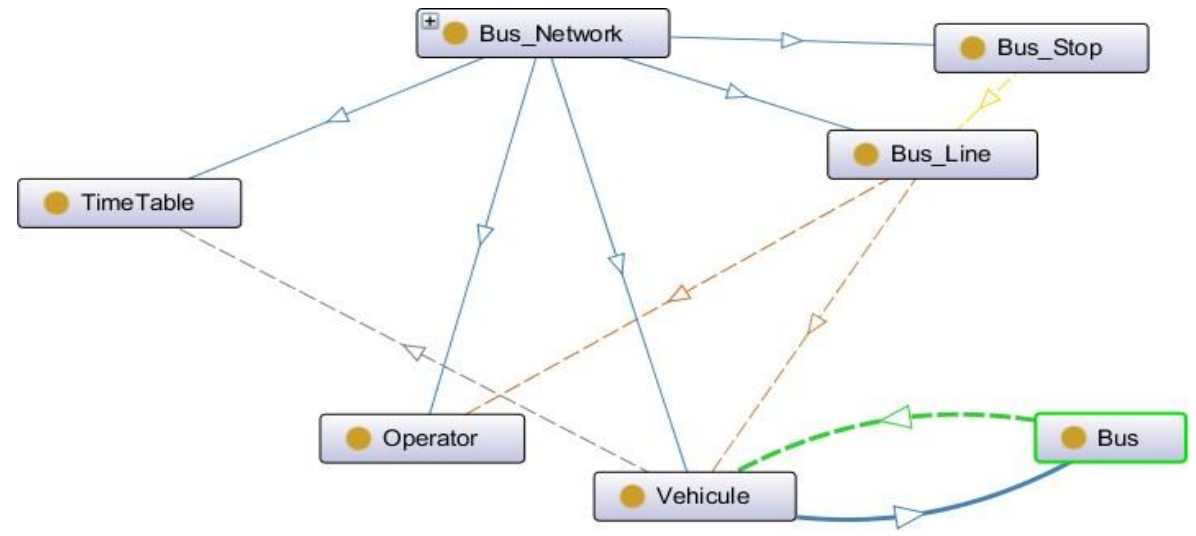

Fig. 6. Bus Network local Ontology

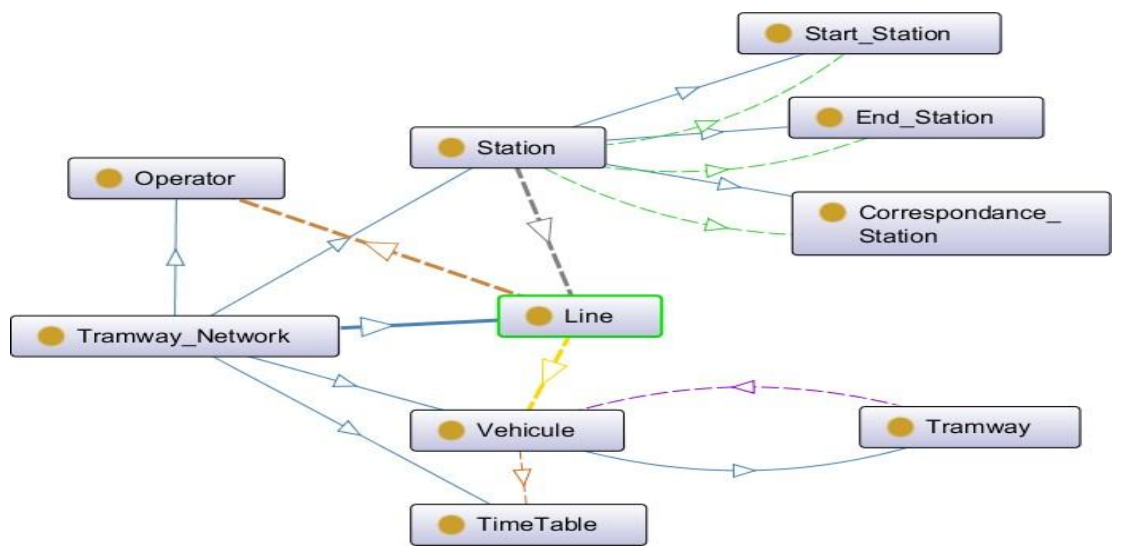

Fig. 7. Tramway Network local Ontology

Now, we focus on the second phase that consists in pulling out the global ontology from different concepts defined in the local ontologies (Tramway network local ontology / Bus Network local ontology).

After analyzing the local ontologies, we have determined the global ontology using OWL Annotation. This Global Ontology called Transportation Network takes up the concepts of the local ontologies and adds new concepts to them in order to meet the need for the multimodal system such as the concept of Transport mode or even Connection Links (Fig.8 and Fig.9).

Moreover, in order to deal with the notion of Safety in the transport field, we defined areas in cities as a concept and we decided to give them notes according to the degree of safety in this zone. In addition, the concept of Travel also joins other concepts of the Global ontology and has as attributes: cost, duration, Start Point and End 


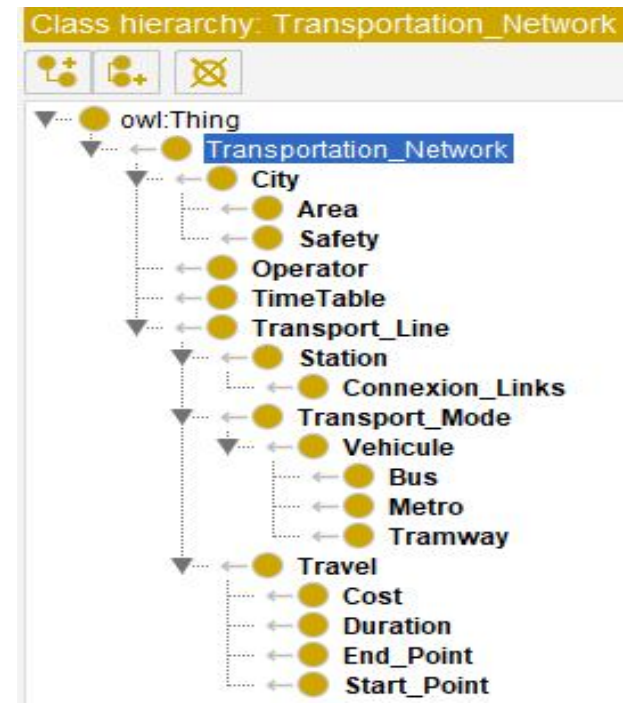

Figure 8: Fragment of Transportation Network Global Ontology

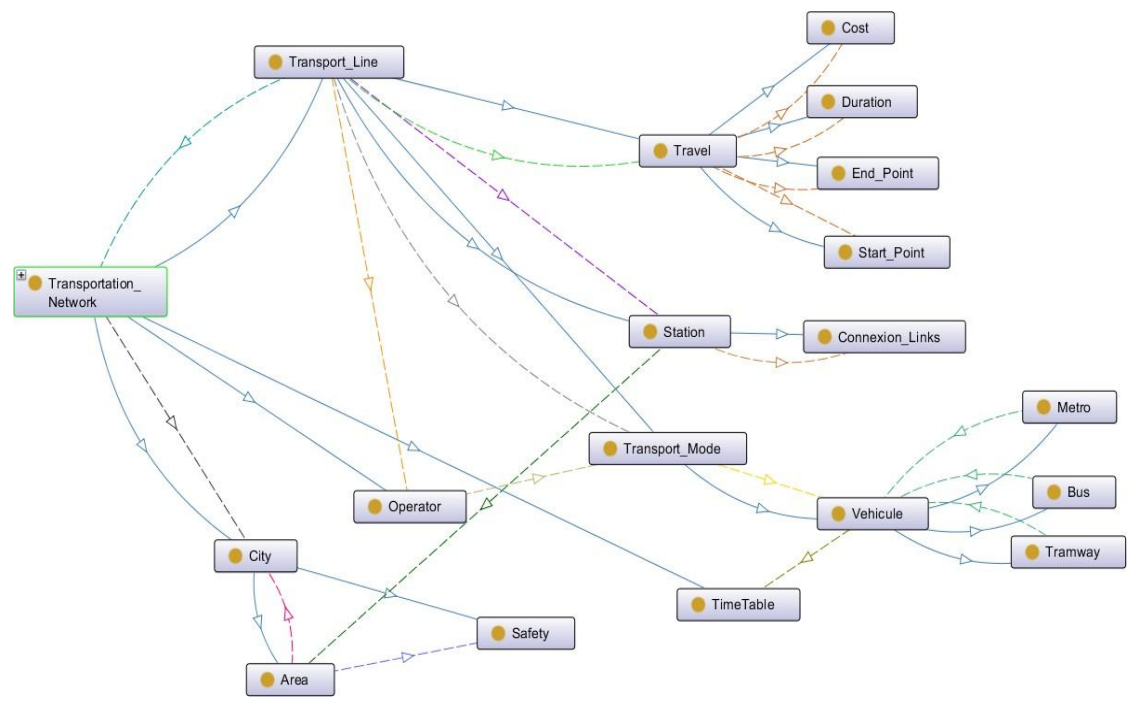

Figure 9: Transportation Network Global Ontology

Finally, we present the third phase that consists in defining the mapping between global and local ontologies using OWL annotation proprieties. This latter allows classes, properties, individuals also the ontology itself, to be annotated with five, pre-defined properties. These properties are illustrated via the Protégé OWL Classes view in Fig.10 and presented in Table1.

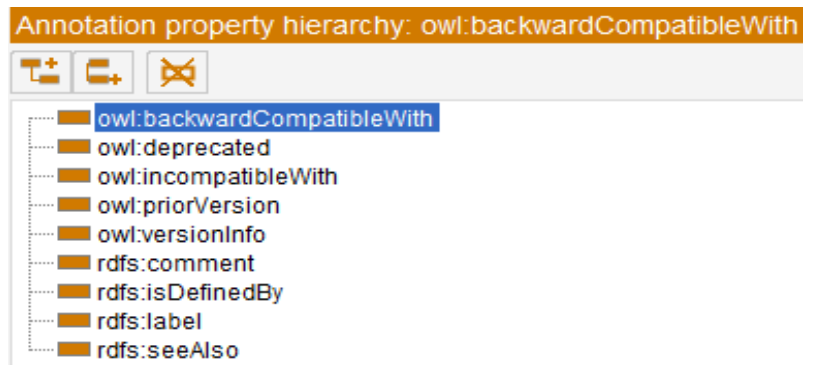

Figure 10: OWL annotation properties illustrated via Protégé
Table 1: The five, pre-defined annotations in OWL.

\begin{tabular}{lll} 
Property & Typing & Purpose \\
\hline owl:versionInfo & String & $\begin{array}{l}\text { OWL version } \\
\text { information }\end{array}$ \\
rdfs:label & String & $\begin{array}{l}\text { Meaningful, } \\
\text { human-readable names }\end{array}$ \\
rdfs:comment & String & Additional information \\
rdfs:seeAlso & URI & $\begin{array}{l}\text { Identify related } \\
\text { resources }\end{array}$ \\
rdfs:isDefinedBy & URI & Reference an ontology \\
\hline
\end{tabular}




\section{CONCLUSION}

In this article, we focus on the semantic layer of our agent-based multi-modal information system. The purpose of this layer is to manage the different sources of data and to build a flexible data model that can be understood by all the agents in the system. In order to deal with this problem, an ontology for the transport system was proposed. This ontology has been developed by understanding the common terms used in the transport system and by looking at the different preference criteria chosen by the user such as cost, duration, number of connections and safety. To build this ontology, we opted for a hybrid architecture, which consists in developing a global ontology from local ontologies. For the present case, we are taking into account only two information resources: Tramway and Bus Data Bases. In a future work, we propose to extend our ontology in order group all the sources so that these data are comprehensible and accessible for the agents of the system. Moreover, we will detail, the negotiation and communication part between the agents themselves and between the different layers of the system. The ontology can be used with a data integration technique to build a multimodal transport system with an agent based semantic approach.

\section{REFERENCES}

1. C. Comtois and B. Slack. The geography of transport systems. Routledge, 2009

2. M. Becker and S. F. Smith. An ontology for multi-modal transportation planning and scheduling. Technical Report CMUR I-TR-98-15, 1997.

3. S. Timpf, Ontologies of wayfinding: a traveler's perspective. Networks and spatial economics, 2(1):9-33, 2002.

https://doi.org/10.1023/A:1014563113112

4. Junli Wang, Zhijun Ding, and Changjun Jiang. An ontology-based public transport query system. In Semantics, Knowledge and Grid, 2005. SKG'05. First International Conference on, pages 62-62. IEEE,2005

5. A. Sadeghi Niaraki and K. Kim. Ontology based personalized route planning system using a multi-criteria decision making approach. Expert Systems with Applications, 36(2):2250- 2259, 2009. https://doi.org/10.1016/j.eswa.2007.12.053

6. H. Mnasser, M. Khemaja, K. Oliveira, and M. Abed. A public transportation ontology to support user travel planning. In Research Challenges in Information Science (RCIS), 2010 Fourth International Conference on, pages 127-136. IEEE, 2010.

7. K. M. De Oliveira, F. Bacha, H. Mnasser, and M. Abed. Transportation ontology definition and application for the content personalization of user interfaces. Expert Systems with Applications, 40(8):3145-3159, 2013.

https://doi.org/10.1016/j.eswa.2012.12.028
8. F. Giunchiglia and B. Dutta. Dera: A faceted knowledge organization framework. Technical report, University of Trento, 2011.

9. F. Giunchiglia, B. Dutta, and V. Maltese. From knowledge organization to knowledge representation. Technical report, Universit a di Trento, 2013.

10. S. Das and F. Giunchiglia. Geoetypes: Harmonizing diversity in geospatial data. In OTM Confederated International Conferences" On the Move to Meaningful Internet Systems", pages 643-653. Springer, 2016.

11. B. Dutta, D. Nandini, and G. Kishore Shahi. Mod: metadata for ontology description and publication. In International Conference on Dublin Core and Metadata Applications, pages 1-9, 2015.

12. C. Masolo, S Borgo, A. Gangemi, N Guarino, A. Oltramari, and L Schneider. Dolce: a descriptive ontology for linguistic and cognitive engineering. WonderWeb Project, Deliverable D17 v2, 1:75- 105, 2003.

13. M. A Musen. The protégé project: a look back and a look forward. AI matters, 1(4):4-12, 2015. https://doi.org/10.1145/2757001.2757003

14. H. ZGAYA, and S. HAMMADI, Multi-Agent Information System Using Mobile Agent Negotiation Based on a Flexible Transport Ontology, In Proceedings of the 2007 International Conference on Autonomous Agents and Multi-agent Systems (AAMAS'2007). May 14-18, Honolulu, Hawaii, 2007.

15. K. Basak, R. Seshadri, L. Azevedo, C. M., 2018. SimMobility - Integrated Simulation Platform. Research project. Massachusetts Institute of Technology, Cambridge, MA, USA [Accessed: 31-Aug-2018].

16. A.S. Niaraki, K. Kim, Ontology based personalized route planning system using a multi-criteria decision making approach, Expert Systems with Applications, 36, pp. 2250- 2259. (2007)

https://doi.org/10.1016/j.eswa.2007.12.053

17. J. LARIOUI, A. EL BYED, A Multi-Agent Information System Architecture For Multimodal Transportation, Embedded Systems and Artificial Intelligence Proceedings of ESAI 2019, Fez, Morocco, Pages 795-803

18. P.Mulholland, 1999, Introduction to ontologies, Version 2, Internal Report, code: RichODL-OU-3/1999, Enriching ODL by knowledge sharing for collaborative computer- based modeling and simulation.

19. F. Lhafiane, A. Elbyed, M. Bouchoum, Reverse Logistics Information Management Using Ontological Approach, World Academy of Science, Engineering and Technology International Journal of Information and Communication Engineering Vol:9, No:2, 2015.

20. J. LARIOUI, A. EL BYED, An Advanced Intelligent Support System for Multimodal Transportation Network Based on Multi-Agent Architecture, 
Advanced Intelligent Systems for Applied Computing Sciences, Volume 4 - Pages 98-106,2020.

https://doi.org/10.1007/978-3-030-36674-2_10

21. A. Ghasempour et al., Using Traffic Control Scheme In Intelligent Transportation System, International Journal of Advanced Trends in Computer Science and Engineering, 8(1.4), 2019, 165- 172

https://doi.org/10.30534/ijatcse/2019/2581.42019

22. Nur Raidah Rahim et al., Integrated Ontology Development for Clinical Decision Support System in the Case Study of Methadone Maintenance Therapy, International Journal of Advanced Trends in Computer Science and Engineering, 8(1.6), 2019, 272 - 282

https://doi.org/10.30534/ijatcse/2019/4181.62019 